This item was submitted to Loughborough's Research Repository by the author.

Items in Figshare are protected by copyright, with all rights reserved, unless otherwise indicated.

\title{
Nonlinear waves in a bi-layer and coupled Klein-Gordon equations
}

PLEASE CITE THE PUBLISHED VERSION

LICENCE

CC BY-NC-ND 4.0

\section{REPOSITORY RECORD}

Khusnutdinova, Karima R.. 2019. "Nonlinear Waves in a Bi-layer and Coupled Klein-gordon Equations". figshare. https://hdl.handle.net/2134/461. 


\section{NONLINEAR WAVES IN A BI-LAYER AND COUPLED KLEIN-GORDON EQUATIONS}

Presented at the IUTAM Symposium "Asymptotics, Singularities and Homogenisation in Problems of Mechanics", July 8-11, 2002, The University of Liverpool, UK

Karima R. Khusnutdinova

Department of Mathematical Sciences, Loughborough University, Loughborough LE11 3TU, UK

and

Institute of Mechanics, Ufa Branch of the Russian Academy of Sciences,

Karl Marx Str. 6, Ufa 450000, Russia

K.Khusnutdinova@lboro.ac.uk

Abstract A system of coupled Klein-Gordon equations is suggested to model onedimensional nonlinear wave processes in a bi-layer. The type of coupling depends on the type of the interface and constitutes an arbitrary element of the Lie group classification problem, which is solved for these equations. The classification results are used to find conservation laws and particular invariant solutions.

\section{INTRODUCTION}

After the intensive study of nonlinear wave processes in recent decades, it has become clear that the same equations, such as, for example, KdV, NLS, etc., appear in many different physical situations (see, for example, $[1,2]$ and the references therein). Since heterogeneity constitutes an essential feature of many physical problems, it makes sense to try to find some simple mathematical models which allow us to study peculiarities of nonlinear wave processes in heterogeneous media.

A possible way to derive such continuum models is to consider the long-wave dynamics of discrete models. One of the most famous models of this type is the Frenkel-Kontorova model [3]. Proposed initially to describe dislocations in metals, it has found numerous applications, and has been generalized in order to describe different phenomena (see the review [4]). The original FK model deals with the situation when an additional semi-infinite plane of atoms is inserted into a perfect crystal lattice. The atoms of the "interface" layer are treated as a one-dimensional 
chain subjected to an external periodic potential produced by the surrounding atoms. For the long waves, in dimensionless variables, one can obtain the integrable sine-Gordon equation whose kink and antikink solutions give the approximate description of the lattice behavior in the vicinity of the dislocation core (see [4]).

In the FK model, one part of the crystal is treated as rigid and motionless. The natural generalization of this model is that of coupled chains of particles [5], i.e., of two one-dimensional periodic chains with linear links between elements and non-linear interaction between the chains. The long wave dynamics of this system is described in dimensionless variables by coupled Klein-Gordon equations

$$
u_{t t}-u_{x x}=f_{u}(u, w), \quad w_{t t}-c^{2} w_{x x}=f_{w}(u, w),
$$

where $c$ is the ratio of the acoustic velocities of the non-interacting components, and $f(u, w)$ describes the interaction between the chains.

Although originating as a generalization of the FK model, coupled Klein-Gordon equations (1.1) can also be considered, depending on the choice of the function $f(u, w)$, as a long-wave limit of a simple lattice model for one-dimensional nonlinear wave processes in a bi-layer, similar to the lattice models proposed by Slepyan and his coworkers in connection with crack propagation in composites (see, for example, [6]). In that case the model parameters are determined by the type of the interface and materials forming the bi-layer. It is also worth noting that the same equations describe some processes in the DNA double helix [7] (see also [8] and the references therein).

In all these models the function $f(u, w)$ should be found experimentally, and therefore, its analytic form is not unique. It is known that the existence of a sufficiently large group of symmetries allows a certain analytic investigation of properties of the equations (see, for example, $[9,10])$. Thus, the problem of group classification of coupled KleinGordon equations (1.1) naturally arises in connection with the model discussed above.

Equations of the type (1.1) with $c=1$ (and arbitrary functions of $u$ and $w$ on the right-hand side) were studied in [11], where the cases admitting Lie-Bäcklund symmetries were picked out, and completely or partially integrable examples were presented. If $f_{u w}(u, w)=0$, the system (1.1) splits into two independent Klein-Gordon equations, whose group classification was given by S.Lie [12] (see also [13]). The results of the Lie group classification of equations (1.1) for $c \neq 1, f_{u w}(u, w) \neq 0$ are presented in this paper, and are used to find conservation laws and to construct particular invariant solutions. In conclusion we discuss some possible applications of this model. 


\section{LIE POINT SYMMETRIES}

The classification is given up to the following equivalence transformations (which preserve the differential structure of equations and change only the arbitrary element, see, for example, [9])

$\tilde{t}=\alpha t+\beta, \quad \tilde{x}=\alpha x+\gamma, \quad \tilde{u}=\lambda u+\mu, \quad \tilde{w}=\lambda w+\nu, \quad \tilde{f}=\frac{\lambda^{2}}{\alpha^{2}} f+\rho$,

where $\alpha, \beta, \gamma, \lambda, \mu, \nu$, and $\rho$ are arbitrary constants and $\alpha \lambda \neq 0$;

$$
\begin{aligned}
& t \rightarrow-t, \quad x \rightarrow x ; \quad t \rightarrow t, \quad x \rightarrow-x \\
& u \rightarrow-u, \quad w \rightarrow w ; \quad u \rightarrow u, \quad w \rightarrow-w \\
& t \rightarrow t, \quad x \rightarrow \frac{x}{c}, \quad u \rightarrow w, \quad w \rightarrow u, \quad c \rightarrow \frac{1}{c}
\end{aligned}
$$

(Groups admitted by equations related by equivalence transformations are similar).

The generator of the admitted Lie point group is sought in the form

$$
X=\xi^{1} \frac{\partial}{\partial t}+\xi^{2} \frac{\partial}{\partial x}+\eta^{1} \frac{\partial}{\partial u}+\eta^{2} \frac{\partial}{\partial w} .
$$

Using the methods of the group analysis of differential equations (see $[9,10])$, the coordinates of the generator can be found as follows:

$$
\begin{aligned}
& \xi^{1}=C_{1} t+C_{2}, \quad \xi^{2}=C_{1} x+C_{3}, \\
& \eta^{1}=C_{4} u+\phi(t, x), \quad \eta^{2}=C_{4} w+\chi(t, x),
\end{aligned}
$$

where functions $\phi(t, x)$ and $\chi(t, x)$ satisfy equations

$$
\begin{aligned}
& \phi_{t t}-\phi_{x x}=\left(2 C_{1}-C_{4}\right) f_{u}+\left(C_{4} u+\phi\right) f_{u u}+\left(C_{4} w+\chi\right) f_{u w}, \\
& \chi_{t t}-c^{2} \chi_{x x}=\left(2 C_{1}-C_{4}\right) f_{w}+\left(C_{4} u+\phi\right) f_{u w}+\left(C_{4} w+\chi\right) f_{w w} .
\end{aligned}
$$

Here $C_{i}, i=\overline{1,4}$ are arbitrary constants.

It follows from $(1.3)$ that when $f(u, w)$ is an arbitrary function, equations (1.1) admit 2-dimensional algebra with the basis

$$
X_{1}=\frac{\partial}{\partial t}, \quad X_{2}=\frac{\partial}{\partial x}
$$

and extension of the admitted algebra (1.4) is possible in those cases when function $f(u, w)$ satisfies equation

$$
\left(a_{1} u+a_{2}\right) f_{u}+\left(a_{1} w+a_{3}\right) f_{w}+a_{4} f=a_{5} u+a_{6} w+a_{7}
$$

where $a_{i}, i=\overline{1,7}$ are constant coefficients. 
For the analysis of the classifying relation (1.5) we first find how its coefficients transform under the equivalence transformations. Then, we find all solutions of equation (1.5) up to equivalence transformations. Finally, solving the determining equations (1.3) for all obtained cases we find the generators additional to (1.4). The results of group classification are presented in Table 1.

\section{Comments on Table:}

- $A, B, C, D$, and $\sigma$ are arbitrary constants, $\delta$ is positive real constant, $\tilde{\delta}$ is nonnegative real constant; $\varepsilon= \pm 1, \tilde{\varepsilon}=0, \pm 1$.

- Constants $\lambda$ and $\mu$ take real or imajinary values.

- $\psi(t, x)=\frac{1}{\delta\left(c^{2}-1\right)}\left\{\left[A \delta c^{2}+B\left(1+\delta^{2} c^{2}\right)\right] t^{2}+\left[A \delta+B\left(1+\delta^{2}\right)\right] x^{2}\right\}$.

- In subcases the group of equivalence transformations can be wider than in general case. For example, if $f(u, w)=F(\delta u-w)+A u$, where $F^{\prime \prime \prime}(z) \neq 0$, additional transformations have the form:

$$
\tilde{u}=u+\theta t x+\omega t+\tau x, \quad \tilde{w}=w+\delta(\theta t x+\omega t+\tau x)
$$

with arbitrary constants $\theta, \omega$, and $\tau$;

$$
\tilde{u}=u+\frac{c^{2} t^{2}+x^{2}}{2\left(c^{2}-1\right)}(\tilde{A}-A), \quad \tilde{w}=w+\frac{c^{2} t^{2}+x^{2}}{2\left(c^{2}-1\right)} \delta(\tilde{A}-A),
$$

where $\tilde{A}$ satisfies equation $\frac{d \tilde{A}}{d a}=\Phi(\tilde{A}, c, \delta),\left.\tilde{A}\right|_{a=0}=A$;

$$
\begin{aligned}
& \tilde{u}=u+\frac{\kappa(A, c, \delta)}{2 \delta\left(c^{2}-1\right)}\left[\left(1+\delta^{2} c^{2}\right) t^{2}+\left(1+\delta^{2}\right) x^{2}\right], \\
& \tilde{w}=w+\frac{\kappa(A, c, \delta)}{2\left(c^{2}-1\right)}\left[\left(1+\delta^{2} c^{2}\right) t^{2}+\left(1+\delta^{2}\right) x^{2}\right], \\
& \tilde{f}=f+\kappa(A, c, \delta)(\delta u-w) .
\end{aligned}
$$

Here $\Phi$ and $\kappa$ are arbitrary functions of their arguments. In accordance with these transformations one may suppose in case under consideration $A=B=0$.

- Additional generators for intersecting subcases are given only once. 
Table 1 Lie group classification of equations (1.1) $\left(c^{2} \neq 1, f_{u w} \neq 0\right)$.

\begin{tabular}{|c|c|}
\hline$f(u, w)$ & Admitted generators \\
\hline Arbitrary function & $X_{1}=\frac{\partial}{\partial t}, \quad X_{2}=\frac{\partial}{\partial x}$ \\
\hline $\begin{array}{l}u^{\sigma} F\left(\frac{u}{w}\right), \quad \sigma \neq 0, \\
\text { if } \sigma \neq 2, \quad F(z) \neq \varepsilon\left(\delta-\frac{1}{z}\right)^{\sigma} \\
\text { if } \sigma=2, \quad F(z) \neq A+\frac{B}{z^{2}}+\frac{1}{z}\end{array}$ & $\begin{aligned} & X_{3}=(\sigma-2)\left(t \frac{\partial}{\partial t}+x \frac{\partial}{\partial x}\right)- \\
& 2\left(u \frac{\partial}{\partial u}+w \frac{\partial}{\partial w}\right)\end{aligned}$ \\
\hline $\begin{array}{l}\exp u F(\tilde{\delta} u-w) \\
F(z) \neq \varepsilon \exp z\end{array}$ & $X_{3}=t \frac{\partial}{\partial t}+x \frac{\partial}{\partial x}-2\left(\frac{\partial}{\partial u}+\tilde{\delta} \frac{\partial}{\partial w}\right)$ \\
\hline $\begin{array}{l}F\left(\frac{u}{w}\right)+\tilde{\varepsilon} \ln u \\
F(z) \neq \tilde{\varepsilon} \ln \left(\delta-\frac{1}{z}\right)\end{array}$ & $X_{3}=t \frac{\partial}{\partial t}+x \frac{\partial}{\partial x}+u \frac{\partial}{\partial u}+w \frac{\partial}{\partial w}$ \\
\hline $\begin{array}{l}F(\delta u-w)+A u w+(\varepsilon-\delta A) \frac{u^{2}}{2}, \\
F^{\prime \prime \prime}(z) \neq 0 \text {. Here if } \\
\text { a) } \lambda^{2}=\frac{\delta \varepsilon-A}{\delta\left(1-c^{2}\right)} \neq 0, \\
\qquad \mu^{2}=\frac{\delta \varepsilon c^{2}-A}{\delta\left(1-c^{2}\right)} \neq 0 \\
\text { b) } \lambda=0, \quad \mu^{2}=-\frac{A}{\delta}=-\varepsilon ; \\
\text { c) } \lambda^{2}=\frac{A}{\delta c^{2}}=\varepsilon, \quad \mu=0\end{array}$ & $\begin{aligned} & X_{i}= \phi_{i}(t, x)\left(\frac{\partial}{\partial u}+\delta \frac{\partial}{\partial w}\right), \\
& i=\overline{3,6} \\
& \phi_{3}= \cos \lambda x \cos \mu t, \phi_{4}=\sin \lambda x \sin \mu t, \\
& \phi_{5}=\cos \lambda x \sin \mu t, \phi_{6}=\sin \lambda x \cos \mu t ; \\
& \phi_{3}=x \cos \mu t, \quad \phi_{4}=\cos \mu t \\
& \phi_{5}=x \sin \mu t, \quad \phi_{6}=\sin \mu t \\
& \phi_{3}=t \cos \lambda x, \quad \phi_{4}=\cos \lambda x \\
& \phi_{5}=t \sin \lambda x, \quad \phi_{6}=\sin \lambda x\end{aligned}$ \\
\hline $\begin{array}{l}F(\delta u-w)+\varepsilon u w-\delta \varepsilon \frac{u^{2}}{2}, \\
F^{\prime \prime \prime}(z) \neq 0, \\
\lambda^{2}=\frac{\varepsilon}{\delta\left(c^{2}-1\right)}\end{array}$ & $\begin{array}{l}X_{i}=\phi_{i}(t, x)\left(\frac{\partial}{\partial u}+\delta \frac{\partial}{\partial w}\right), i=\overline{3,6} \\
\phi_{3}=\cos \lambda x \cos \lambda t, \quad \phi_{4}=\sin \lambda x \sin \lambda t, \\
\phi_{5}=\cos \lambda x \sin \lambda t, \quad \phi_{6}=\sin \lambda x \cos \lambda t\end{array}$ \\
\hline
\end{tabular}

\section{INVARIANT SOLUTIONS AND CONSERVATION LAWS}

The results of classification allow us to find conservation laws and construct particular invariant solutions (self-similar, traveling waves, etc.) 
Table 1 (continued)

Lie group classification of equations $(1.1)\left(c^{2} \neq 1, f_{u w} \neq 0\right)$.

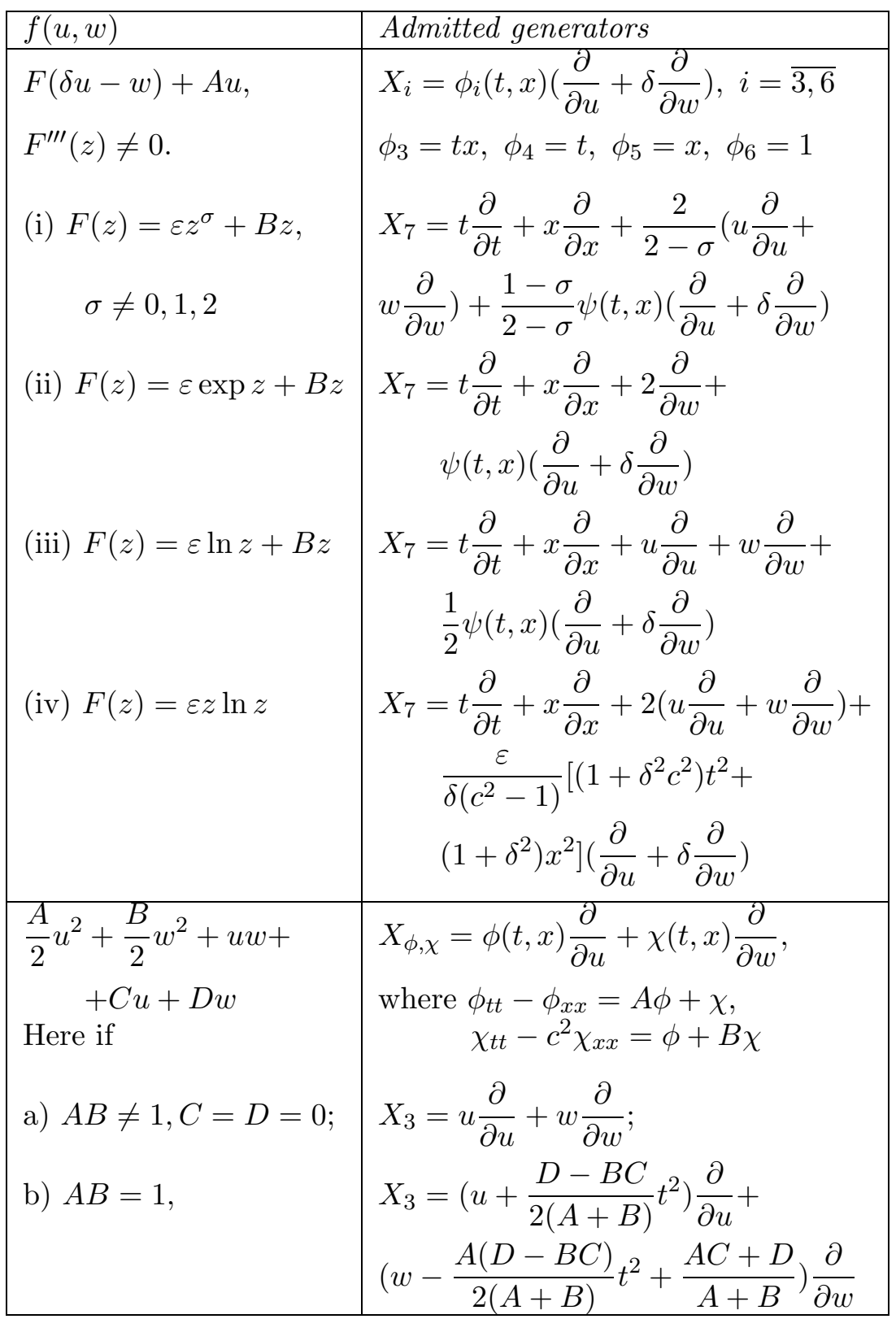

The system (1.1) with an arbitrary function $f(u, w)$ can be formulated by means of Lagrangian principle from the density

$$
L=\frac{1}{2}\left(u_{t}^{2}+w_{t}^{2}-u_{x}^{2}-c^{2} w_{x}^{2}\right)+f(u, w) .
$$


Indeed,

$$
\frac{\delta L}{\delta u}=f_{u}(u, w)-u_{t t}+u_{x x}=0, \frac{\delta L}{\delta w}=f_{w}(u, w)-w_{t t}+c^{2} w_{x x}=0
$$

give us equations (1.1). Therefore, knowing infinitesimal operators (1.2) and using the Nöther theorem (see [10]), one can find conservation laws

$$
D_{t} A^{1}+D_{x} A^{2}=0
$$

where $A^{i}=L \xi_{\alpha}^{i}+\left(\eta^{\alpha}-\xi^{k} u_{k}^{\alpha}\right) \frac{\partial L}{\partial u_{i}^{\alpha}}, \alpha=1,2, i=1,2$; and $u^{1}=u, u^{2}=$ $w ; u_{1}^{\alpha}=u_{t}^{\alpha}, u_{2}^{\alpha}=u_{x}^{\alpha}$. The operators (1.4) are admitted with any function $f(u, w)$. The corresponding conservation laws for energy and momentum have the form

$$
\begin{aligned}
& D_{t}\left[\frac{1}{2}\left(u_{t}^{2}+w_{t}^{2}+u_{x}^{2}+c^{2} w_{x}^{2}\right)-f(u, w)\right]-D_{x}\left[u_{t} u_{x}+c^{2} w_{t} w_{x}\right]=0, \\
& D_{t}\left[u_{t} u_{x}+w_{t} w_{x}\right]-D_{x}\left[f(u, w)+\frac{1}{2}\left(u_{t}^{2}+w_{t}^{2}+u_{x}^{2}+c^{2} w_{x}^{2}\right)\right]=0 .
\end{aligned}
$$

In those cases where the dimension of the admitted algebra is bigger than 2 , there are additional conservation laws, which can be easily written down explicitly.

Let us also find some particular invariant solutions of equations (1.1) for the case $f(u, w)=\cos (\delta u-w)$. Introducing $\tilde{u}=\delta u, \tilde{w}=w$ as new dependent variables we rewrite equations as

$$
u_{t t}-u_{x x}=-\delta^{2} \sin (u-w), \quad w_{t t}-c^{2} w_{x x}=\sin (u-w)
$$

(the tildes are omitted). Although this case is not the most beneficial from the point of view of admitted symmetries (6-dimensional Lie algebra), it is interesting as a possible generalization of the FK model. Here, $\delta^{2}=m_{2} / m_{1}$ has a meaning of the ratio of masses of particles in the "lower" and the "upper" chains. For $\delta^{2} \rightarrow 0$, setting $u=0$, we obtain for $w$ the sin-Gordon equation, being the long-wave approximation of the FK model. Thus, the FK model appear as a natural limit of the coupled equations (1.9).

Equations (1.9) admit generator $\frac{\partial}{\partial t}+v \frac{\partial}{\partial x}+\alpha t\left(\frac{\partial}{\partial u}+\frac{\partial}{\partial w}\right)$, where $v$ is an arbitrary constant, $\alpha$ may be supposed nonnegative constant. The solution which is invariant with respect to this generator for $v^{2} \neq 1, c^{2}$, and $\frac{1+\delta^{2} c^{2}}{1+\delta^{2}}$ has the form

$$
u=\sigma^{-1}\left[\delta^{2}\left(v^{2}-c^{2}\right) p(x-v t)+\eta\right], w=\sigma^{-1}\left[\left(1-v^{2}\right) p(x-v t)+\eta\right]
$$




$$
\begin{aligned}
& \text { where } \quad \sigma=\delta^{2}\left(v^{2}-c^{2}\right)+v^{2}-1, \\
& \eta=\alpha\left(1+\delta^{2}\right) v t x-\frac{\alpha}{2}\left[\left(1+\delta^{2} c^{2}\right) t^{2}+\left(1+\delta^{2}\right) x^{2}\right]+A(x-v t)+B \\
& p^{\prime \prime}=-\lambda \sin p-a, \\
& \lambda=\frac{\delta^{2}\left(v^{2}-c^{2}\right)+v^{2}-1}{\left(v^{2}-1\right)\left(v^{2}-c^{2}\right)}, \quad a=\frac{\alpha\left(1-c^{2}\right)}{\left(v^{2}-1\right)\left(v^{2}-c^{2}\right)} .
\end{aligned}
$$

Here the prime denotes derivative with respect to the argument of the function; $A$ and $B$ are arbitrary constants, let us suppose $A=B=0$.

If $\lambda<0$, then introducing $\tilde{p}=p+\pi$ equation (1.11) can be rewriten in the form $\tilde{p}^{\prime \prime}=-|\lambda| \sin \tilde{p}-a$. Therefore, analyzing (1.11) one can suppose $\lambda>0$. The energy integral of (1.11) may be written in the form $\left(p^{\prime}\right)^{2}+2 \lambda(1-\cos p)+2 a p-2 E^{*}=2 E, \quad E^{*}=\lambda-\sqrt{\lambda^{2}-a^{2}}-a \arcsin \frac{a}{\lambda}$.

If the condition $|a|<\lambda$ is satisfied, then any value of $E$ from the region

$$
0<E<2 \lambda-\pi|a|-2 E^{*}
$$

defines a bounded periodic solution of (1.11) describing nonlinear oscillations near the point of equilibrium $\quad p=-\arcsin \frac{a}{\lambda} \quad$ in the region $p \in\left[p_{1}, p_{2}\right]$, where $p_{1}$ and $p_{2}$ are the smallest and the largest roots of the equation

$$
\lambda(1-\cos p)+a p-E^{*}=E
$$

on the interval $]-\pi+\arcsin \frac{a}{\lambda}, \pi+\arcsin \frac{a}{\lambda}[$. It is assumed here that $\left.\arcsin \frac{a}{\lambda} \in\right]-\frac{\pi}{2}, \frac{\pi}{2}[$.

If $\alpha=0$, equation (1.11) turns out to be the mathematical pendulum oscillations equation which is integrable in elliptic functions (see, for example, [14]). In this case solutions of the system (1.9) describing periodic waves have the form:

$$
u=2 U \arcsin \{k \operatorname{sn}[\sqrt{\lambda}(x-v t), k]\}=W w
$$

— "fast" waves, propagating with velocities $\left.\quad v^{2} \in\right] S, M[\cup] L,+\infty[$, where $S=\min \left\{1, c^{2}\right\}, M=\frac{1+\delta^{2} c^{2}}{1+\delta^{2}}, L=\max \left\{1, c^{2}\right\}$,

$$
u=2 U \arcsin \{\operatorname{dn}[\sqrt{|\lambda|}(x-v t), k]\}=W w,
$$


— "slow" waves, propagating with velocities $v^{2} \in[0, S[\cup] M, L[$. Here

$$
U=\frac{\delta^{2}\left(v^{2}-c^{2}\right)}{\delta^{2}\left(v^{2}-c^{2}\right)+v^{2}-1}, \quad W=\frac{\delta^{2}\left(v^{2}-c^{2}\right)}{1-v^{2}}, \quad 0<k<1 .
$$

In the $k=1$ limiting case "slow" periodic waves become solitary waves:

$$
u=4 U \arctan \{\exp \sqrt{|\lambda|}(x-v t)\}=W w .
$$

Using the fact that Eqs.(1.9) admit reflections

$$
t \rightarrow-t, \quad x \rightarrow x ; \quad t \rightarrow t, \quad x \rightarrow-x ; \quad u \rightarrow-u, \quad w \rightarrow-w ;
$$

one can obtain solutions with other combinations of signs.

If $\delta \rightarrow 0\left(m_{1} \gg m_{2}\right)$, the solitary waves may propagate with the velocities $v^{2} \in\left[0, c^{2}[\right.$. In that case the displacement of particles is independent on the velocity of wave propagation. If the masses $m_{1}$ and $m_{2}$ are comparable, the solitary waves may propagate with the velocities $v^{2} \in[0, S[\bigcup] M, L[$. Therefore, if the acoustic velocities of noninteracting components are different $\left(c^{2} \neq 1\right)$, a gap appears in the velocity spectrum of the solitary waves, i.e. the system acts as a kind of a filter of solitary waves. Here the relative displacement ("upper" particles relative to the "lower" ones) remains the same as in the FK model (per period of the chain), but the absolute displacement depends on the velocity of the wave.

Solutions for the nonlinear waves in a presence of additional shear forces acting either on both chains in the opposite directions or on just one chain can be constructed using (1.10) with $\alpha \neq 0$ and the equivalence transformations (1.6) - (1.8), which were found above. Using other admitted generators we can find some other invariant solutions.

\section{CONCLUSION}

The simple lattice-based model proposed here can be used for studying the peculiarities of propagation and interaction of one-dimensional nonlinear waves in a bi-layer. It can be useful for problems related to energy exchange between the layers, long-short wave resonance, delamination, etc. The model can be modified to take into account other degrees of freedom by considering chains of interacting mechanical dipoles [15] instead of chains of point masses. These topics will be discussed elsewhere.

\section{Acknowledgments}

I would like to thank I.Sh.Akhatov, V.A.Baikov, D.E.Pelinovsky and V.V.Silberschmidt for useful discussions. 


\section{References}

[1] Dodd, R K, Eilbek, J C, Gibbon, J D, and Morris, H C (1984) Solitons and Nonlinear Wave Equations. London: Academic Press Inc.

[2] Scott, A (1999) Nonlinear Science: Emergence and Dynamics of Coherent Structures. New York: Oxford University Press Inc.

[3] Kontorova, T A, and Frenkel, Ya I (1938) On the theory of plastic deformation and twinning I, Zh. Eksp. Teor. Fiz. 8 (1) 89 - 95.

[4] Braun, O M and Kivshar, Yu S (1998) Nonlinear dynamics of the Frenkel-Kontorova model, Phys. Rep. 306 (1998) 1-108.

[5] Akhatov, I Sh, Baikov, V A, and Khusnutdinova, K R (1995) Nonlinear dynamics of coupled chains of particles, J. Appl. Maths Mechs 59 (3) 353 - 361.

[6] Slepyan, L I (1998) Some Basic Aspects of Crack Dynamics. In: Chrepanov, G P ed. (1998) Fracture. A Topical Encyclopedia of Current Knowledge. Malabar, Florida: Krieger Publishing Co.

[7] Yomosa, S (1983) Soliton excitations in deoxiribonucleic acid (DNA) double helices, Phys. Rev. A 27 (4) 2120 - 2125.

[8] Yakushevich, L V (1998) Nonlinear Physics of DNA. Chichester: Wiley.

[9] Ovsiannikov, L.V. (1982) Group Analysis of Differential Equations. New York: Academic Press.

[10] Olver, P (1986) Applications of Lie Groups to Differential Equations. New York: Springer-Verlag.

[11] Zhiber, A V, Ibragimov, N H, and Shabat, A B (1979) Equations of Liouville type, Dokl. Akad. Nauk SSSR. 249 (1) 26 - 29.

[12] Lie, S. (1881) Discussion der Differentialgleichung $\frac{\partial^{2} z}{\partial x \partial y}=F(z)$, Arch. für Math. og Naturv. 6 (1) 112 - 124.

[13] Ibragimov, N H ed. (1994) CRC Handbook of Lie Group Analysis of Differential Equations, Vol. 1, Symmetries, exact solutions and conservation laws. Boca Raton: CRC Press.

[14] Kamke, E (1967) Differentialgleichungen. Lösungsmethoden und Losungen. Teil 1, 8th ed. Leipzig: Akademics Verlag, Greest \& Portig.

[15] Khusnutdinova, K R (1992) Nonlinear Waves in a Double Row Particle System, Vestn. MGU, 1 (2) 71-76. 